\title{
METODOLOGIA HORIZONTAL DE ENSINO APLICADA A CONCEPÇÃO E PROTOTIPAGEM DE PLACA DE CIRCUITO IMPRESSO DE UM MODULADOR FM
}

DOI: 10.37702/2175-957X.COBENGE.2021.3382

Guilherme Buriol - guilhermeburioll@gmail.com

Universidade Federal de Santa Maria

Rodolfo Behr 450

97105-440 - Santa Maria - RS

Laira Milena Moraes Tomé - laira.moraes12@gmail.com

Universidade Federal de Santa Maria

Rua Otacílio de Castro 11

- Santa Maria - RS

Natanael Rodrigues Gomes - natanael-rodrigues.gomes@ufsm.br

Universidade Federal de Santa Maria

UFSM, Avenida Roraima 1000

97105-970 - Santa Maria - RS

Davi Sehnem Castro - davisehnem99@gmail.com

Universidade Federal de Santa Maria

Doutor Bozano 303

97015-001 - Santa Maria - RS

Emmanuel Adamski de Moura - emmanomoura@gmail.com

Universidade Federal de Santa Maria

Rua Ernesto Pereira 698

97105-140 - Santa Maria - RS

Resumo: Possuir conhecimento sobre a concepção de placas de circuito impresso, desde a formação do projeto em software até a soldagem dos componentes, é de suma importância para a formação de todo engenheiro. Dessa forma, o Programa de Educação Tutorial Engenharia Elétrica (PET-EE) da Universidade Federal de Santa Maria (UFSM) elaborou e ministrou um minicurso com o objetivo de oferecer oportunidades de aprendizado, teórica e prática, para os alunos de diversos cursos 
da universidade neste conteúdo. O minicurso foi ministrado no decorrer de três dias, em que cada dia uma parte diferente da concepção da placa era abordada. $O$ circuito escolhido foi um Modulador FM, já que esse permitia o contato com componentes eletrônicos básicos, possibilitando que pessoas sem experiência pudessem acompanhar o projeto, auxiliando-os na expansão de seus conhecimentos nas áreas da eletrônica e das telecomunicações. No final do minicurso, todos os alunos conseguiram concluir e testar o seu circuito ao sincronizar o aparelho de rádio na frequência projetada, o que permitiu aprimorar seus conhecimentos no uso de equipamentos de laboratório, como osciloscópio e analisador de espectro. Além de desenvolver habilidades práticas de confecção de placas de circuito impresso nos ouvintes, o minicurso também permitiu aprimoramento da didática por parte dos ministrantes, visto que estes também eram alunos do curso de engenharia elétrica.

Palavras-chave: Circuito impresso. Modulador FM. Programa Educação Tutorial. Minicurso. 


\section{METODOLOGIA HORIZONTAL DE ENSINO APLICADA A CONCEPÇÃO E PROTOTIPAGEM DE PLACA DE CIRCUITO IMPRESSO DE UM MODULADOR FM}

\section{INTRODUÇÃO}

O Programa de Educação Tutorial do curso de Engenharia Elétrica (PET EE) da Universidade Federal de Santa Maria (UFSM) é um programa do governo federal que incentiva alunos da graduação para atividades de ensino, extensão e pesquisa. Entre as ações realizadas pelo grupo está a Jornada de Minicursos, que é uma atividade realizada anualmente no período de duas semanas no segundo semestre letivo, sendo composto de vários minicursos ministrados tanto pelos próprios membros do PET EE, quanto por outros graduandos que desejam compartilhar seu conhecimento em uma área tecnológica que considera útil.

Tal ato tem como alvo outros estudantes que tenham vontade de aprender sobre os assuntos propostos. Além disso, o dinheiro arrecadado com as inscrições é destinado a projetos sociais sem fins lucrativos ou instituições que necessitam de ajuda, fazendo com que o ouvinte contribua para essas entidades e, ao mesmo tempo, beneficie-se com o conhecimento passado pelo ministrante.

Durante a IX Jornada de Minicursos, foi oferecido o minicurso de Confecção e Prototipagem de Placas de Circuito Impresso, no qual foi desenvolvido um circuito de um Modulador em Frequência Modulada (FM), desde a etapa de projeto até a montagem .O mesmo foi proposto para ser desenvolvido em 3 encontros de 2 horas cada, onde cada ouvinte desenvolveria sua própria placa de circuito impresso (PCB).

\section{JUSTIFICATIVA}

Atualmente, o curso de graduação em engenharia elétrica da Universidade Federal de Santa Maria (UFSM) não possui disciplinas que proporcionem experiências de projeto e confecção de Placas de Circuito Impresso (PCB). Neste âmbito, essa competência normalmente é desenvolvida em atividades extracurriculares, como projetos de pesquisa e extensão. Isso pode ocasionar dificuldades por parte do aluno em desenvolver protótipos de qualidade, visto que a montagem e a prototipagem de um circuito elétrico é uma parte importante em sua concepção, interferindo diretamente no resultado esperado.

Desta forma, o Programa de Educação Tutorial da Engenharia Elétrica (PET-EE) elaborou um Minicurso, em sua IX Jornada de Minicursos, a fim de auxiliar os estudantes de graduação a desenvolver a competência acima citada. O minicurso foi elaborado a partir de metodologias horizontais de ensino, ou seja, de estudante para estudante, possibilitando o aprendizado mútuo entre ouvinte e ministrante.

O circuito escolhido deveria ser simples e compor componentes básicos de eletrônica, para possibilitar a explicação de seu funcionamento, visto que o minicurso era aberto para qualquer pessoa que quisesse se inscrever no mesmo, não necessitando ter 
algum conhecimento de eletrônica básica. Assim, um circuito Modulador FM supriu essa necessidade por conta de sua funcionalidade ser facilmente assimilada pelos ouvintes, bem como proporcionou o uso de ferramentas específicas, como o analisador de espectros.

Este circuito é compacto, o que possibilita sua implementação nos três encontros que foram propostos para a realização dessa atividade. Além disso, os recursos utilizados para a sua montagem eram facilmente encontrados e apresentavam, relativamente, baixo custo, possibilitando a criação de mais vagas para o minicurso.

Ainda, outros motivos levaram a escolha do circuito Modulador FM, como a possibilidade de visualização prática do resultado, o que leva a motivação dos ouvintes quanto a realização das tarefas, bem como auxilia os mesmos na construção e estudo de outras PCBs por conta própria. Isso faz com que os estudantes aperfeiçoem as técnicas utilizadas para a montagem das placas, e talvez, futuramente utilizem estes conhecimentos em âmbito profissional.

\section{OBJETIVOS}

De acordo com as questões apresentadas anteriormente, o minicurso teve como principal objetivo introduzir os alunos nas diferentes etapas de elaboração de uma placa de circuito impresso. A primeira etapa consistiu em entender o funcionamento de softwares de simulação de projetos, aprendendo a buscar e reconhecer os diferentes componentes eletrônicos presentes nas bibliotecas do software, projetar as trilhas da placa de forma a minimizar o espaço usado e onde posicionar cada componente a fim de evitar interferências eletromagnéticas entre os mesmos.

A segunda etapa tratou da impressão do circuito projetado e a passagem deste para as placas de cobre. Nesta etapa, o intuito é mostrar como preparar a placa para a impressão, as diferentes maneiras de realizar a transferência térmica e a corrosão da placa, bem como ensinar a utilizar os equipamentos de segurança e demonstrar a sua importância

$\mathrm{Na}$ terceira etapa foi realizada a soldagem da placa e o teste final de funcionamento. Nela, os alunos praticaram a solda de componentes na placa, a utilização de multímetros para testar o funcionamento do circuito, permitindo identificar curto circuitos, além de serem capazes de resolver possíveis problemas, oriundos de erros nas etapas anteriores, e realizar os devidos testes com o analisador de espectros para verificar a funcionalidade do modulador.

Além do aprendizado técnico proporcionado por cada etapa, o minicurso também tem como objetivo permitir um modelo de aprendizado interativo entre os estudantes, onde estes são incentivados a trocar conhecimento e experiências adquiridas no decorrer dos encontros que possam ser úteis durante a confecção da placa de circuito impresso.

\section{METODOLOGIA}

O circuito utilizado para este minicurso foi constituído por um microfone de eletreto, que possui a função de converter a voz em sinais elétricos correspondentes. Além disso, o projeto possui um estágio amplificador, montado com um Transistor de Junção Bipolar $\mathrm{BC} 547 \mathrm{~B}$, a fim de aumentar a amplitude do sinal elétrico gerado pelo microfone. Em 
seguida, o sinal passa por um circuito modulador em frequência (FM), no qual a frequência de onda do sinal de voz é modificada por uma frequência de portadora, resultando em um sinal modulado com frequência elevada, a fim de permitir sua transmissão através de uma pequena antena, contida na PCB.

Para realizar a modulação FM, foi utilizado um arranjo contendo um Transistor de Junção Bipolar, BC547B e um circuito ressonante formado por um indutor e um capacitor (LC), que possui a função de gerar o sinal de portadora de alta frequência. Após o estágio modulador, o sinal passa por um Transistor 2N2907B, disposto de forma a elevar a corrente do sinal, para que a transmissão possa ser feita em distâncias maiores. Em seguida, o sinal modulado é transmitido pela antena contida na placa.O diagrama esquemático pode ser visualizado na imagem a seguir:

Figura 1: Circuito Modulador FM utilizado para o desenvolvimento do Minicurso.

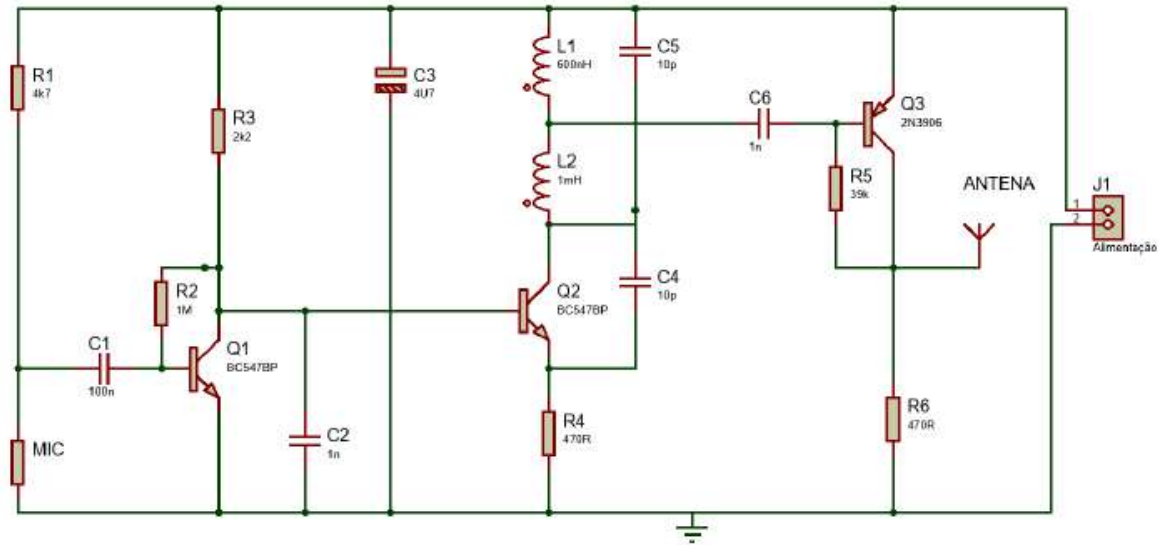

Fonte: Acervo próprio

Para que o Modulador FM funcione corretamente, é necessário que os componentes do circuito LC estejam corretamente dimensionados, visto que a frequência do sinal de portadora ( $f$ portadora $)$ é definida pela frequência natural de ressonância ( $\omega_{n}$ ) do tanque LC. Desta forma:

$$
\begin{aligned}
& \omega_{n}=\frac{1}{\sqrt{L C}} \\
& f_{\text {portadora }}=\frac{1}{2 \pi \sqrt{L C}}
\end{aligned}
$$

Para gerar a frequência natural de ressonância do circuito LC, foram utilizados dois capacitores, de $10 \mathrm{pF}$ associados em série, a fim de gerar um resultado de $5 \mathrm{pF}$. Além disso, foi utilizado um indutor bobinado manualmente, contendo aproximadamente 5 milímetros de diâmetro e 4 voltas. Uma vez que a bobinagem do indutor foi realizada manualmente, não se pôde averiguar uma medida exata e padronizada para sua indutância.

Como foi utilizado um aparelho de rádio para fazer a demodulação e captação do sinal, a frequência de portadora deveria ficar entre os valores máximos e mínimos 
captados pelo rádio para sinais FM, que, para o aparelho utilizado, era de $87 \mathrm{MHz}$ a 108 $\mathrm{MHz}$. Assim, para possibilitar ajustes na frequência de portadora, o indutor foi construído utilizando uma derivação central conectada a um dos capacitores do circuito LC, permitindo ajustar a frequência sem a necessidade de substituir componentes. A utilização de uma associação de capacitores em série ao invés de um único capacitor de $5 p F$ também foi realizada com este objetivo. Caso fossem necessárias alterações bruscas na frequência da onda portadora, uma associação de capacitores permite alcançar uma maior gama de valores se comparado com as capacitâncias comerciais para um componente.

O circuito definido foi então previamente montado pelos membros do projeto. Com o uso de um analisador de espectros a frequência de sinal de portadora foi estimada em $92 \mathrm{MHz}$. A partir deste valor, conhecendo a capacitância do circuito LC, pôde-se calcular a indutância do tanque, através da equação (2), encontrando $600 \mathrm{nH}$.

Com o valor encontrado para a frequência de portadora, foi dimensionada a antena a ser utilizada no projeto. Para o teste do circuito, a antena foi confeccionada a partir de um fio de cobre de $1 \mathrm{~m}$ de comprimento. Para o dimensionamento da antena, foi adotado o seguinte equacionamento, onde $\lambda$ representa o comprimento de onda do sinal e $c$ representa a velocidade da luz no vácuo:

$$
\begin{aligned}
& \lambda=\frac{c}{f_{\text {portadora }}} \\
& l_{\text {antena }}=\frac{\lambda}{4}
\end{aligned}
$$

A partir de equação (3), foi possível concluir que o comprimento de onda da frequência portadora foi de 3,26 metros. Assim, considerando a equação (4) para dimensionamento de antena dipolo, resultou em uma antena de 81,5 centímetros de comprimento. Com o dimensionamento dos componentes concluído, estes foram separados em kits distribuídos aos alunos para facilitar e agilizar a montagem.

\subsection{Planejamento e implementação:}

Para projetar e implementar o circuito proposto em placa de circuito impresso, o minicurso foi dividido em três aulas. A primeira aula ocorreu em um laboratório de informática, no qual fora instalado previamente o software de simulação escolhido para a atividade. Como alguns dos participantes ainda não haviam cursado as disciplinas de eletrônica e de teoria de circuitos elétricos, foi necessário fazer uma explicação simplificada do funcionamento de transistores e da ressonância gerada pelo circuito LC. $\mathrm{Na}$ sequência, os alunos iniciaram a montagem do circuito apresentado no software de projetos de PCB, onde foi possível realizar a simulação do mesmo, verificando a concordância com a teoria previamente apresentada. Em seguida, os ministrantes apresentaram algumas dicas e boas práticas de roteamento de componentes em PCBs, a fim de auxiliar os participantes a desenhar as trilhas de cobre que conectam os componentes do circuito. Ao final do encontro, com o projeto finalizado, foi gerado um 
arquivo imprimível do circuito final, conforme pode ser visualizado na Figura 2, além de uma simulação em 3D do circuito, conforme Figura 3.

Figura 2: Circuito construído em software simulador

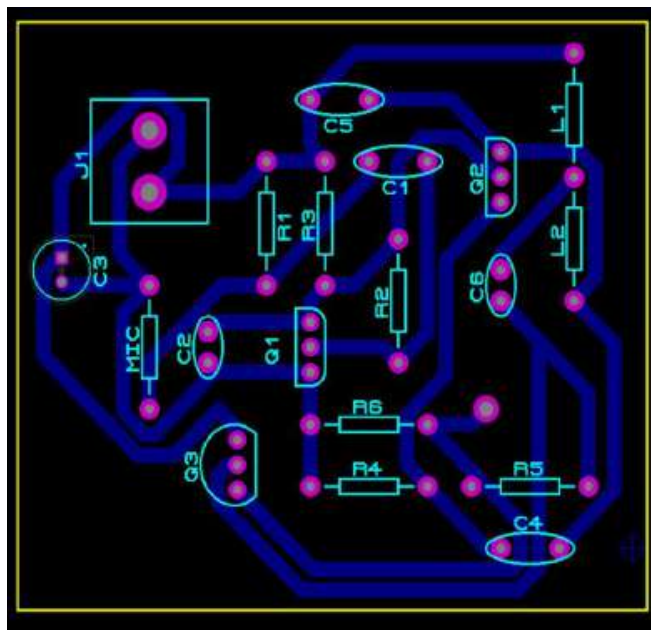

Fonte: Acervo próprio

Figura 3: Visualização em 3D do circuito projetado.

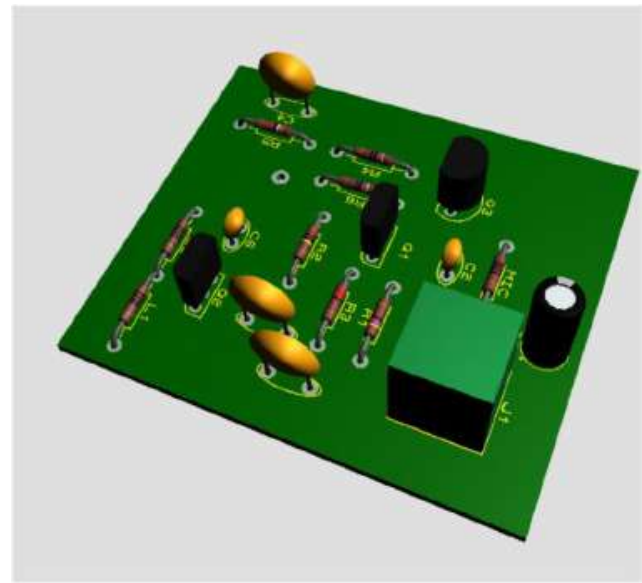

Fonte: Acervo próprio

Para a segunda aula, primeiramente tratou-se das especificações necessárias para a transferência térmica e a corrosão da placa. Assim, foram passadas informações a respeito do tipo de papel e tinta a ser usada para a impressão, além de alguns métodos que podem ser utilizados para a corrosão das placas. Em seguida, foram abordadas curiosidades a respeito do processo industrial de produção de PCBs, com caráter apenas informativo.

O segundo tópico da aula constituiu-se da limpeza das placas de fenolite e a transferência térmica. Esta última foi feita utilizando um ferro de passar roupas como fonte de calor. Nesta etapa, o processo foi inicialmente demonstrado pelos ministrantes. Após, cada aluno realizou a transferência térmica em sua placa.

A corrosão das placas foi realizada a partir de uma mistura de peróxido de hidrogênio 10 volumes, obtido em farmácia e ácido clorídrico, obtido em loja de material de construção na forma de produto de limpeza de obras. Este método foi escolhido pois 
não utiliza o percloreto de ferro, decisão tomada com o intuito de informar aos alunos a existência de um método diferente de corrosão, mais barato e com resultados semelhantes. Foram também fornecidos aos participantes máscaras e luvas, como equipamento de proteção individual necessário. Na sequência, realizou-se a perfuração das placas, além de envernizá-las, para garantir maior durabilidade e resistência à oxidação.

No terceiro encontro, nos primeiros minutos foram apresentadas informações técnicas e procedimentos corretos de soldagem com estanho. Então, os ministrantes auxiliaram os alunos na montagem das placas. Conforme estes finalizavam, os membros do PET-EE os auxiliavam na fase de testes do circuito. O circuito foi testado primeiramente através de um Analisador de espectros. Com este aparelho, é possível identificar a faixa de frequência da portadora, averiguando se o circuito está emitindo algum sinal modulado em frequência, dentro do limite que o aparelho de rádio consegue demodular. Para alguns alunos, este teste bastou, mostrando que o circuito estava funcionando corretamente, sendo possível sintonizar o aparelho de rádio na frequência de portadora indicada pelo analisador de espectro.

Para outros alunos, o circuito precisou de ajustes em sua faixa de frequências para poder ser demodulado pelo aparelho de rádio. Nestes casos, os ministrantes auxiliaram na regulagem da derivação do indutor, a fim de modificar a frequência de portadora do circuito. Em outros casos, foram cometidos erros de soldagem, que foram detectados com multímetros. Estes defeitos serviram como forma de transmitir para os ouvintes noções práticas de testes de circuitos, a fim de identificar e consertar defeitos, que são comuns em prototipagem de circuitos.

\section{RESULTADOS}

Com o objetivo de avaliar a metodologia utilizada durante o curso, bem como a didática dos ministrantes, foi elaborado um questionário avaliativo que foi enviado para os alunos participantes após o último encontro. Foram obtidas seis respostas de um total de oito participantes. O Gráfico 1, referente à primeira pergunta do questionário, indaga os participantes sobre o quão adequada foi a distribuição dos processos de confecção entre as três aulas ministradas.

Gráfico 1 - Feedback dos alunos Questão 1.

Você acredita que a forma como as aulas foram divididas (software, impressão/corrosão e soldagem) foi:

6 respostas

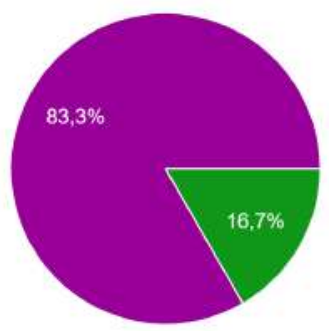


Foi possível observar que 5 dos seis alunos consideraram a distribuição das aulas Muito Boa, e uma pessoa a classificou como Boa. Esses números mostram que a distribuição foi adequada aos participantes, de forma a abordar uma parcela suficiente de conteúdo em cada encontro, sem sobrecarregá-los com novas informações.

Uma das justificativas apresentadas para a realização do minicurso era a falta de disciplinas na universidade que proporcionam este tipo de atividade. Dessa forma, a pergunta 3, quantificada no Gráfico 2, verificou o nível de conhecimento prévio dos participantes sobre cada conteúdo abordado.

Gráfico 2 - Feedback dos alunos Questão 3.

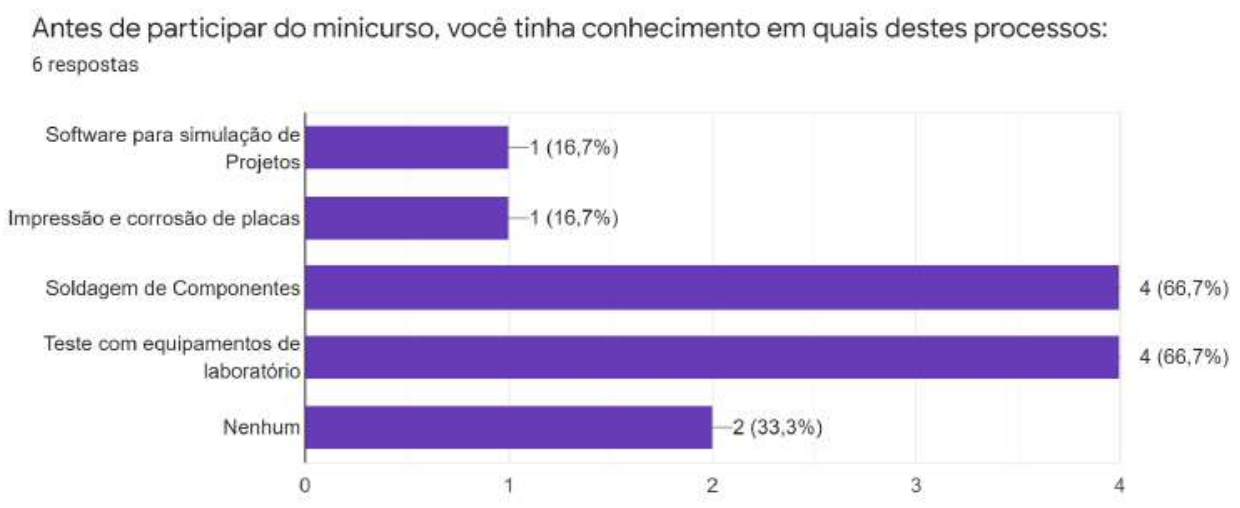

Fonte: Acervo próprio

Pode-se observar que a maior parte dos participantes não tinham nenhum conhecimento no uso de software de simulação de projetos ou impressão e corrosão de placas. Nota-se ainda que dois alunos afirmaram não possuir conhecimento em nenhum dos processos apresentados. Analisando ainda o resultado da pergunta 4 (Gráfico 3), que questiona quais dos processos os alunos mais adquiriram novos conhecimentos, percebe-se que todas as aulas foram proveitosas, mas em especial a referente a impressão e corrosão de placas de circuito impresso, onde todos os alunos afirmaram receber novas informações. 
Gráfico 3 - Feedback dos alunos Questão 4.

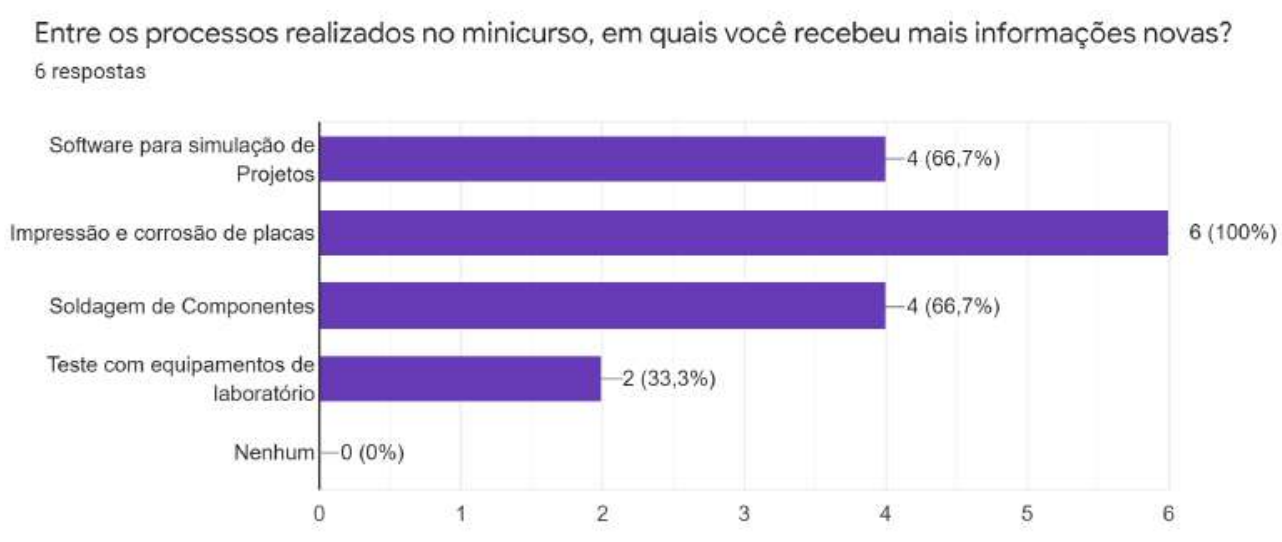

Fonte: Acervo próprio

Um dos objetivos do minicurso era proporcionar um aprendizado de maneira horizontal, de aluno para aluno. Para verificar o quão positivo é este método, foi elaborada a pergunta 2 (Gráfico 4), onde 0 se refere a "Nada Positivo" e 5 a "Muito Positivo"

Gráfico 4 - Feedback dos alunos Questão 2.

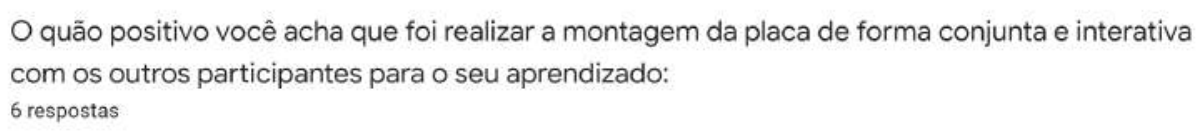
6 respostas

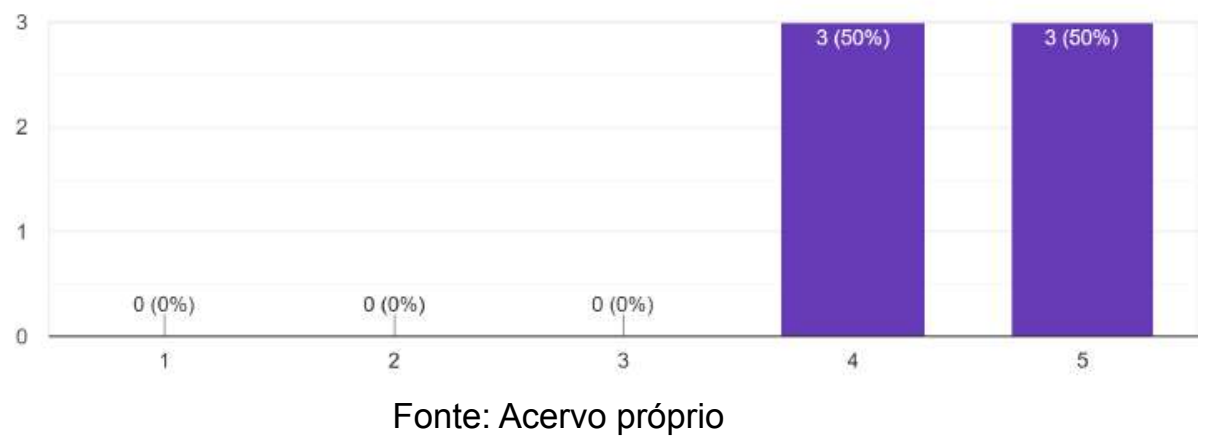

Percebe-se que $100 \%$ dos participantes marcaram as opções 4 e 5 , o que mostra um elevado nível de aprovação por parte dos alunos em relação a ter uma aula interativa e com certa independência do auxílio do professor. De modo geral os objetivos do minicurso foram alcançados. O conhecimento técnico desenvolvido ajudou muitos alunos que tinham dúvida e interesse no assunto, além do aprendizado que os ministrantes desenvolveram ao interagir e trocar experiências enquanto procuravam transmitir o conteúdo.

\section{CONSIDERAÇÕES FINAIS}

Após a finalização do minicurso, os membros do grupo PET-EE puderam analisar os benefícios e a viabilidade da realização de outras edições deste projeto. Assim, foi 
possível averiguar que o minicurso foi de grande valia tanto para os que participaram como ouvintes, quanto para os alunos que participaram como ministrantes. Durante as aulas, notou-se que os ouvintes demonstraram bastante interesse em adquirir conhecimentos de forma prática, ao realizar atividades como a solda dos componentes, corrosão da placa e testes no circuito. Observou-se, também, que alguns participantes já tinham conhecimento prévio sobre o tema, e mesmo assim, puderam aprimorá-lo, pois foram apresentados diferentes métodos para a realização das distintas etapas, bem como suas vantagens e desvantagens.

Por parte dos ministrantes, o minicurso promoveu o desenvolvimento de questões relacionadas à comunicação interpessoal, como didática e oratória. Além disso, também foi consolidado o conhecimento técnico a respeito do tema tratado, possibilitando responder diversos questionamentos que surgiram por parte dos ouvintes no decorrer do curso.

\section{REFERÊNCIAS}

ALL DATASHEET. Fair Child BC547 Datasheet. Disponível em: https://pdf1.alldatasheet.com/datasheet-pdf/view/50730/FAIRCHILD/BC547.html. Acesso em 12 de novembro de 2019.

ALL DATASHEET. STM Microeletronics 2N2907 Datasheet. Disponível em: https://pdf1.alldatasheet.com/datasheet-pdf/view/21679/STMICROELECTRONICS/2 N2907.html. Acesso em 12 de novembro de 2019.

BASSAN, G. M.; CUSTÓDIO, L. P. Tutorial confecção de placas de circuito impresso pelo processo de fototransferência. Curso de engenharia elétrica - UTFPR. Curitiba. Outubro de 2014.

\section{DESTRO II. Tutorial completo sobre transferência térmica e corrosão de $\mathrm{PCl}$. Disponível \\ em:}

http://destro.todavia.com.br/tutorial_transferencia-corrosao-daniel-bonadio.php. Acesso em 12 de novembro de 2019.

HOMEMADE CIRCUITS. Home Made Walkie-talkie Circuit. Disponível em: https://www.homemade-circuits.com/homemade-walkie-talkie-circuit/. Acesso em 12 de novembro de 2019.

QSL. Modulação Angular: FM E PM. Disponível em: https://www.qsl.net/py4zbz/teoria/fmpm.htm. Acesso em 12 de novembro de 2019.

\section{CONCEPTION AND PROTOTYPING OF A FM MODULATOR PRINTED CIRCUIT BOARD DEVELOPED IN THE FORM OF A STUDENT TAUGHT MINI-COURSE}

Abstract: Having knowledge about the making of printed circuit boards, from the design of the project on software to the welding of the components, is of paramount importance for the training of every engineer. Thus, the Programa de Educação Tutorial Engenharia 
Elétrica (PET-EE) of the Universidade Federal de Santa Maria (UFSM) designed and taught a mini-course with the objective of offering learning opportunities, both theoretical and practical, for students of several university courses in this subject. The mini-course was taught in the span of three days, each one discussing a different part of the board design. The chosen circuit was an FM Modulator, witch only required basic electronic components, allowing people with no experience means to follow the project, helping them to expand their knowledge in the fields of electronics and telecommunications. At the end of the mini-course, all students were able to complete and test their circuit by synchronizing the radio device at the projected frequency, which allowed them to improve their knowledge in the use of laboratory equipment, such as the oscilloscope and spectrum analyzer. In addition to developing the listeners practical skills in making printed circuit boards, the mini-course also granted the teachers a opportunity to improve their tutoring skills, since they were also students in the electrical engineering course.

Keywords: Printed Circuit. FM Modulator. Programa Educação Tutorial. Mini-course. 